\title{
Representaciones sociales sobre actividad física y educación física en docentes bogotanos ${ }^{*}$
}

\author{
Diana Alexandra Camargo Rojas* \\ Linda Moreno Zabaleta**
}

Recibido: diciembre 13 de 2016 - Aceptado: octubre 3 de 2019

\section{Resumen}

En contextos escolares, la educación física se reconoce como el escenario para la promoción de la actividad física (AF); no obstante, en algunos colegios, los docentes amplían los conceptos de la educación física tradicional hacia otras formas de movimiento. Por esta razón, el presente estudio busca comprender las representaciones sociales de docentes bogotanos frente a la actividad física y la educación física. El presente estudio tiene un paradigma interpretativo, con un enfoque cualitativo y un diseño narrativo. Se realizaron entrevistas a profundidad a nueve docentes de la localidad de Suba y seis docentes de la localidad de Ciudad Bolívar. La información se registró en matrices comparativas, cuyo contenido fueron los discursos

"Artículo de investigación vinculado al proyecto Representaciones sociales y prácticas de actividad física en docentes de educación física, Localidad de Suba UPZ 71 desarrollado por Yuri Natali Ortega Jiménez en la Universidad del Rosario y a la tesis denominada Imaginarios sociales de los profesores de educación física de la UPZ 68 de la Localidad de Ciudad Bolívar, referente a la actividad física, la educación física y el cuerpo desarrollada por Edwin Gualtero Jara en la Universidad Santo Tomás. Financiación propia. Grupo kinesiología, salud y desarrollo, Universidad Nacional de Colombia, Bogotá, Colombia. Citar como: Rojas, D y Moreno, L. (2019). Representaciones sociales sobre actividad física y educación física en docentes bogotanos. Revista de Investigación Cuerpo, Cultura y Movimiento, 9(1), 115-135. DoI: https:// doi.org/10.15332/2422474x/5356

"* Magíster en Salud Pública, profesora asistente, Universidad Nacional de Colombia, Bogotá, Colombia. Correo electrónico: dacamargor@unal.edu.co / ORCID: https://orcid.org/0000-0003-0922-1603.

*** Profesional de Cultura Física, Deporte y Recreación, Universidad Santo Tomás, Bogotá, Colombia. Correo electrónico: moonblue0509@gmail.com / ORCID: 00000003-1195-4590 
descritos por los docentes de acuerdo con las categorías y subcategorías establecidas. Esta investigación arrojó que las representaciones sociales de los docentes de educación física entrevistados tienen varios aspectos en común; con respecto a la actividad física, manifiestan que su práctica es fundamental para el desarrollo físico, emocional y social de las personas. Desde las representaciones, la educación física se relaciona con la salud, pero en la práctica se continúan implementando estrategias basadas en el deporte y la higiene corporal.

Palabras clave: representaciones sociales, educación física, actividad motora, escuela. 


\section{Social representations on physical activity and physical education in teachers from Bogota}

Abstract

In school contexts, physical education is recognized as the setting for the promotion of physical activity (PA). However, in some schools, teachers extend the concepts of traditional physical education to other forms of movement. For this reason, this study seeks to understand the social representations of teachers from Bogota in relation to physical activity and physical education. The present study has an interpretive paradigm with a qualitative approach and a narrative design. In-depth interviews were conducted to nine teachers from Suba and six teachers from Ciudad Bolívar. The information was recorded in comparative matrices, whose content were the discourses described by the teachers according to the established categories and subcategories. This research showed that the social representations of the physical education teachers interviewed have several aspects in common; with regards to physical activity, they state that their practice is fundamental for physical, emotional and social development of people. From the representations, physical education is related to health, but in practice, strategies based on sports and body hygiene continue to be implemented.

Keywords: social representations, physical education, motor activity, school. 


\section{Introducción}

La construcción de una representación social propia de la educación física y de la actividad física por parte de los docentes puede ser compleja, ya que, al ser una percepción generada por la experiencia y la cultura, presenta diferentes significados. Es así como nace la necesidad de encontrar esas diferencias y similitudes que permitan generar una representación social en el marco educativo dentro del Distrito Capital.

De esta manera, la educación física se relaciona constantemente con la escuela y se enfoca principalmente en el aspecto pedagógico; es decir, en lo que se aprende y, luego, en lo que sabe hacer el estudiante en cuanto a movimientos específicos de algún tipo de expresión corporal (deporte, danza o actividad física en general). Asimismo, se relaciona muy poco con la salud, ya que no se presenta como un generador de hábitos de vida saludable.

En este marco, la Universidad Nacional de Colombia realizó un estudio sobre las representaciones sociales que tienen los docentes de una localidad en Bogotá sobre la actividad física, en donde se encontró una estrategia orientada a la inclusión de la actividad física en la educación escolar, que estaba dirigida a diferentes instituciones educativas. Esta propuesta fue impulsada por el Instituto Distrital para la Recreación y el Deporte (IDRD), desde el año 2002, a través de su programa Muévete Escolar. Dicho programa tiene como objetivo movilizar a toda la comunidad educativa en torno a la importancia de la práctica regular de actividad física y la sana alimentación, teniendo en cuenta que los hábitos establecidos durante la infancia y la adolescencia tienden a mantenerse en la vida adulta (Ariza, Laverde y Parraga, 2013, p. 13).

Por otro lado, Ruiz (2009), en su artículo El pensamiento docente sobre los procesos de enseñanza en la educación física, devela el pensamiento del docente de educación física frente a su labor educativa. Ruiz cita a Carretero (1993), quien afirma lo siguiente:

[...] el profesor al igual que el alumno, no copia la información de la realidad, sino que va realizando un proceso de interiorización progresiva mediante sucesivas acomodaciones, asimilaciones y equilibraciones, en consecuencia, la enseñanza se encuentra mediatizada por ideologías y pensamientos que hacen que el docente de educación física, asuma 
posturas y se identifique con tendencias de enseñanza y por qué no, con una forma de vida. (Ruiz, 2009, p. 80)

Por otra parte, según lo manifiesta Cerezo (2004), la formación inicial de los licenciados en Ciencias de la Actividad Física y el Deporte está orientada a distintos campos profesionales: la docencia, el entrenamiento deportivo, la gestión deportiva y las actividades recreativas y de tiempo libre. Esto origina que la formación sea la que guíe el desarrollo de las actividades dentro de la enseñanza de la educación física, orientando este aspecto a la experimentación práctica, la adquisición de fundamentos teóricos y la vivencia de experiencias para su utilización en contextos educativos. Por lo tanto, de manera general, podemos decir que el conocimiento que los docentes deben tener sobre la enseñanza de la educación física parte de unos conocimientos básicos y una cultura profesional, más personal y particular del contexto donde se desarrolle la acción educativa (Cerezo, 2004).

Igualmente, Almonacid (2012) realiza el estudio La educación física como espacio de transformación social y educativa: perspectivas desde los imaginarios sociales y la ciencia de la motricidad (p. 177), en el que examina la importancia que tiene la educación física en el aula, al igual que el imaginario que tienen los actores de esta asignatura para la restructuración del área en Chile.

Almonacid (2012) plantea que:

De lo expuesto precedentemente, podemos intentar acercarnos al aula del sub-sector Educación Física desde la noción de imaginario social, puesto que en los más de cien años en que se ha desarrollado la disciplina en el país, se ha instalado una comprensión sobre su sentido, estructura, finalidad, propósitos y proceso didáctico en las diferentes generaciones de estudiantes, constituyendo una representación colectiva de lo que la sociedad chilena entiende y significa como una actividad curricular que se despliega desde los primeros años de escolaridad, a través de continuos procesos de socialización que principian con imágenes que se cargan progresivamente de sentido y significado. (pp. 182-183)

Por otro lado, es importante mencionar que las representaciones sociales pueden variar según el contexto. En el 2009, Ruiz realiza un estudio acerca 
del concepto que tiene el docente sobre la educación física, en el cual menciona que:

Es evidente la evolución que tienen los conceptos y cómo se van construyendo de la mano con los cambios sociales, culturales, políticos, económicos y científicos en las diferentes épocas, por ello, abordar el pensamiento que el docente tiene sobre los procesos de enseñanza de la educación física, implica inicialmente la pregunta por la concepción que se tiene de la misma, cómo se entiende y desde dónde se parte. (p. 81)

Últimamente se han desarrollado diversas investigaciones en este campo, en las cuales se observa el análisis acerca de los imaginarios, conceptos y representaciones que poseen los docentes sobre la actividad física y la educación física. Con esto se evidencia la existencia de diversidad de términos y enfoques de los docentes en cuanto a la actividad física y la educación física, la falta de coherencia entre la práctica pedagógica y el discurso que plantea cada docente.

Estos aspectos conllevan a que la metodología aplicada en la enseñanza de la educación física en los colegios de Bogotá carezca de un direccionamiento claro en cuanto a sus contenidos y, por ende, se desarrollen prácticas con una planeación precaria. Por lo anterior, la presente investigación pretendió comprender las representaciones sociales que construyen los docentes de educación física frente a la educación física y la actividad física en las localidades de Suba y Ciudad Bolívar de la ciudad de Bogotá.

\section{Referentes teóricos}

\section{Educación física}

Existen muchos conceptos que tratan de definir a la educación física; y pareciera que todos los autores plantean su propia concepción defendiendo su posición, la cual, desde la corriente que les parece, es la que describe la función de la educación física y los elementos que la componen de manera más concreta y acertada. También, en cada una de las definiciones se observa una influencia de la cultura y el medio en que estas se desarrollan. A continuación, la tabla 1 presenta un resumen de las definiciones que tienen diversos autores para el concepto de educación física. 
Tabla 1. Definiciones de educación física

Autores

Definición

\section{Federation Internationale $D^{\prime}$ Education Physique} (2000)

Gallo (2010)

Rozengardt (2015)

González et al.
La educación física es la parte de la educación que utiliza de una manera sistemática las actividades físicas y la influencia de los agentes naturales: aire, sol, agua, etc., como medios específicos.

La educación física permite que el cuerpo sea un instrumento perfecto de adaptación del individuo a su medio, tanto físico como social, gracias a la adquisición de la destreza que consiste en ejecutar con precisión el gesto adecuado en cualquier caso particular y que se puede definir como el dominio fisiológico para la adaptación a una situación dada.

Es una tradición muy antigua de la humanidad, tradición de realizar actividades corporales y motrices vinculadas directamente al juego, al ocio, a la preparación para la vida productiva y social, a la realización de hazañas ligadas a rituales diversos, a la concreción de ideales guerreros, a las necesidades de defensa y de dominación, a los valores admitidos en relación con el cuerpo y su puesta en acción.

La educación física se convierte en una pedagogía de las conductas motrices, en la medida que trata de optimizar o mejorar las conductas motrices de los educados. El profesor de educación física se convierte en un experto observador de las conductas motrices de sus alumnos y, una vez catalogadas y sistematizadas, trata de sugerir o plantear las situaciones motrices que provocan la optimización de las conductas observadas en función de un determinado proyecto pedagógico y de aquello que sea de mayor interés y congenien para la persona afectada.

Fuente: elaboración propia.

\section{Actividad física}

Al igual que la educación física, la cual se define desde varios conceptos y genera diferentes tendencias, la actividad física presenta diversidad en la forma que los autores la definen. Inicialmente, se debe tener en cuenta que hoy en día es abordada desde el ámbito de la salud, ya que es tomada como la herramienta principal para la prevención de enfermedades crónicas no transmisibles. A continuación, la tabla 2 presenta un resumen de algunas definiciones del concepto de actividad física. 
Tabla 2. Definiciones de actividad física

Autores

Autores

Organización Mundial de

la Salud (OMS) (2009)

Leal, Aparicio, Luti, Acosta, Finol, Rojas,

Toledo, Cabrera,

Mermúdez y Velasco (2009)
Definición

Todos los movimientos que forman parte de la vida diaria, incluyendo trabajo, recreación, ejercicios y deportes. Es necesaria para la promoción y conservación de la salud.

"La actividad física es la ciencia del movimiento humano, es un estado fisiológico que requiere la movilización y distribución rápida de diversos elementos, para asegurar el suplemento adecuado de energía que permitirá realizar el trabajo muscular”.

Fuente: elaboración propia.

Caspersen, Powell y Christensen, citados por Gualtero y Camargo (2016) mencionaron otros tipos de definiciones, pero una que predomina es entender la actividad física como "cualquier movimiento corporal producido por los músculos esqueléticos y que requiere un cierto gasto energético" (p. 29).

\section{Método}

Esta investigación se fundamenta en el paradigma interpretativo con un enfoque cualitativo. "Los estudios cualitativos se caracterizan por un proceso de construcción interactiva del argumento teórico y la evidencia empírica" (Maxwell, 1996). Se fundamenta en un diseño narrativo, en el cual se comprenden las representaciones a partir de los discursos, percepciones y experiencias de los sujetos. De igual manera, incluye las revisiones de documentos, imágenes y observaciones. Para realizar el contraste entre los discursos, se aplicó el método comparativo, que, según Sartori (como se citó en Toscano, 2011), tiene como objetivo la búsqueda de similitudes y disimilitudes.

\section{Unidad de análisis}

El presente estudio se realizó en docentes de educación física de las localidades de Ciudad Bolívar y Suba de la ciudad de Bogotá. En la localidad 
19 de Ciudad Bolívar, upz 68, se entrevistaron seis profesores de los colegios IED Bicentenario, Minuto de Buenos Aires, JJ Rojas, Antonio García y Sotavento; cinco de los profesores son licenciados en educación física y uno de ellos tiene maestría en Ciencias del deporte. En la localidad de Suba se entrevistaron en total nueve profesores de las IED Gerardo Molina, Tibabuyes Universal y Delia Zapata Olivella. Todos son docentes nombrados en propiedad por la Secretaría de Educación de Bogotá y son licenciados en el área de educación física.

\section{Herramienta de recolección de información}

El instrumento de recolección de datos consistió en entrevistas a profundidad aplicadas a la población. Luego, se realizó un análisis comparativo de las representaciones de los docentes de educación física ubicados en colegios de las UPZ 68 de la localidad Ciudad Bolívar y la uPz 71 de la localidad de Suba.

\section{Resultados y discusión}

Para comprender el contexto en el cual se desarrolló la investigación, a continuación, se enuncian algunas características de las localidades estudiadas. Suba es la localidad número 11 de la ciudad, se encuentra ubicada al noroccidente de la ciudad y es la cuarta localidad más extensa de la capital, con 10056 hectáreas, después de Sumapaz, Usme y Ciudad Bolívar, respectivamente. En términos de actividad física, la localidad de Suba busca ampliar los espacios deportivos en zonas donde tradicionalmente las personas no han contado con la posibilidad de ejercitarse, por la falta de mobiliario apropiado o porque carecen de dinero para acceder a espacios privados, como gimnasios o polideportivos. La estrategia pretende que los ciudadanos, además de utilizar los espacios deportivos de la localidad, adquieran hábitos saludables que diariamente podrán realizar desde su hogar (Secretaría de Cultura Recreación y Deporte, 2015).

La localidad de Ciudad Bolívar cuenta con una población aproximada de 713764 habitantes, según datos del DANE correspondientes al 2005. Está compuesta por grupos indígenas, campesinos, afrodescendientes, entre otros. Ciudad Bolívar está integrada por 360 barrios, con 12998 hectáreas 
de superficie (3433 en zona urbana y 9555 en rural). Al igual que en Suba, en esta localidad se implementan programas que benefician a la comunidad. Para niños y niñas se plantea el desarrollo de planes deportivos organizados de acuerdo con la edad, el crecimiento, la maduración, el desarrollo físico e intelectual, y el medio geográfico y social en el que viven los niños. Esto con el propósito fundamental de desarrollar principios éticos, cívicos y morales, y que a la par adquieran una cultura física que propenda por mejorar su desarrollo físico, social y afectivo, para contribuir de este modo a elevar la calidad de vida (Secretaría de Cultura Recreación y Deporte, 2015).

Teniendo en cuenta estos elementos de contexto, a continuación, se presentan los resultados obtenidos en la presente investigación. En la tabla 3 se observan los resultados de la localidad de Ciudad Bolívar y la tabla 4 presenta los resultados de la localidad de Suba. Se evidencian las categorías de educación física y actividad física, y las subcategorías de percepción, beneficio y práctica.

Tabla 3. Matriz comparativa de la localidad de Ciudad Bolívar

\begin{tabular}{|c|c|c|c|}
\hline Categorías & Subcategorías & Ejes de indagación & Resultado \\
\hline \multirow{3}{*}{ Educación física } & Percepción & $\begin{array}{l}\text { ¿Qué concepto } \\
\text { tiene de la } \\
\text { educación física? }\end{array}$ & $\begin{array}{l}\text { La educación física se considera } \\
\text { como la integración de cuerpo y } \\
\text { movimiento, ciencia de lo corporal, } \\
\text { conocimiento que comprende el } \\
\text { cuerpo, mente y cuerpo, autocuida- } \\
\text { do y salud. }\end{array}$ \\
\hline & Beneficio & $\begin{array}{l}\text { ¿Cuáles beneficios } \\
\text { trae la enseñanza } \\
\text { de la educación } \\
\text { física en los } \\
\text { alumnos? }\end{array}$ & $\begin{array}{l}\text { Los docentes afirman que los bene- } \\
\text { ficios que la educación física le pue- } \\
\text { de brindar al ser humano tienen que } \\
\text { ver netamente con la adquisición de } \\
\text { valores y de hábitos. Por el contra- } \\
\text { rio, es poco lo que aporta a los be- } \\
\text { neficios relacionados con la salud, } \\
\text { aduciendo que la intensidad horaria } \\
\text { no es suficiente para generar un im- } \\
\text { pacto directo sobre la salud. }\end{array}$ \\
\hline & Práctica & $\begin{array}{l}\text { ¿Cómo desarrolla } \\
\text { el aspecto práctico } \\
\text { de la educación } \\
\text { física? }\end{array}$ & $\begin{array}{l}\text { Los docentes, mayoritariamente, } \\
\text { utilizan el deporte en la clase de } \\
\text { educación física como herramienta } \\
\text { para poner en práctica su clase, pero } \\
\text { a su vez utilizan otros medios como } \\
\text { la expresión corporal, el juego y la } \\
\text { lúdica. }\end{array}$ \\
\hline
\end{tabular}




\section{Percepción}

Beneficio

Actividad física
¿Qué entiende por actividad física?

Para usted, ¿qué beneficios trae la práctica de la actividad física?
¿Qué experiencia ha tenido o tiene

Práctica con respecto a la práctica de la actividad física?
Se define la actividad física como todo aquello que implique movimiento y que se puede desarrollar desde diferentes ámbitos como el deporte, ejercicios de caminatas o carreras, o de fortalecimiento muscular.

Los docentes se refieren a tres aspectos básicos relacionados con los beneficios de la actividad física: a nivel psicológico o mental, a nivel fisiológico y a nivel social. Desde lo psicológico, se encuentran variables como sentirse bien o alegre, y el equilibrio mental. Desde lo fisiológico, mejora las capacidades físicas. Desde lo social, facilita la generación de relaciones interpersonales.

Los docentes han tenido una vasta experiencia en la práctica de actividad física y consideran el deporte como la principal herramienta para este fin. También se puede evidenciar que las lesiones se han convertido en un impedimento para poder llevar a cabo cualquier tipo de práctica deportiva o de actividad física en sí.

Fuente: elaboración propia.

En la tabla 3 se evidencia que, según los educadores, los conceptos de actividad física y educación física mantienen una relación entre ellos, ya que por medio de la educación física se realizan actividades que fortalecen el bienestar para los alumnos en cualquier contexto. El concepto de educación física es entendido por los docentes como un constructo que integra cuerpo, movimiento y mente. Ellos comprenden que este trinomio puede ser un vehículo para el bienestar y el autocuidado; no obstante, aunque el concepto parece integrador, las acciones continúan teniendo un enfoque deportivista, y el tiempo de duración y la frecuencia de las clases sigue siendo escasa para cumplir objetivos para la salud. Por otra parte, para los docentes, la actividad física involucra todo movimiento corporal humano, que tiene beneficios 
físicos, psicológicos y sociales. Aunque reconocen estos beneficios, solo emplean el deporte como medio para la práctica de la actividad física en sus clases y esto se asocia con la experiencia corporal de cada uno de ellos.

Tabla 4. Matriz comparativa de la localidad de Suba

\begin{tabular}{|c|c|c|c|}
\hline Categorías & Subcategorías & Ejes de indagación & Resultado \\
\hline \multirow[b]{3}{*}{ Educación física } & Percepción & $\begin{array}{l}\text { ¿Qué concepto } \\
\text { tiene de la } \\
\text { educación física? }\end{array}$ & $\begin{array}{l}\text { La educación física es vista como un } \\
\text { área integral, en la que se pueden } \\
\text { desarrollar múltiples aspectos en el } \\
\text { estudiantado, tanto a nivel corporal } \\
\text { como a nivel social. Sin embargo, } \\
\text { sienten que aún falta reconocimien- } \\
\text { to a nivel institucional. }\end{array}$ \\
\hline & Beneficio & $\begin{array}{l}\text { ¿Cuáles beneficios } \\
\text { trae la enseñanza } \\
\text { de la educación } \\
\text { física en los } \\
\text { alumnos? }\end{array}$ & $\begin{array}{l}\text { Los docentes sí ven a la educa- } \\
\text { ción física como un elemento cla- } \\
\text { ve en la salud de los estudiantes, } \\
\text { pero consideran que sus alcances } \\
\text { no son suficientes al enfrentar } \\
\text { problemas como la limitación } \\
\text { del tiempo de la clase o la esca- } \\
\text { sa cantidad de horas de clase a la } \\
\text { semana. }\end{array}$ \\
\hline & Práctica & $\begin{array}{l}\text { ¿Cómo desarrolla } \\
\text { el aspecto práctico } \\
\text { de la educación } \\
\text { física? }\end{array}$ & $\begin{array}{l}\text { Las prácticas que más realizan los } \\
\text { docentes son las deportivas; en } \\
\text { muchos casos están relacionadas } \\
\text { con las prácticas sociales o for- } \\
\text { mativas que se pueden realizar a } \\
\text { través del deporte y la educación } \\
\text { física. En cuanto a las prácticas de } \\
\text { actividad física que los docentes } \\
\text { desarrollan en menor medida, se } \\
\text { encontró que las habilidades mo- } \\
\text { trices y la condición física tienen } \\
\text { de igual forma un papel impor- } \\
\text { tante para el estudiantado. En el } \\
\text { caso de la condición física, esta } \\
\text { se encontró como categoría emer- } \\
\text { gente, ya que se hace necesaria en } \\
\text { el mejoramiento de la calidad de } \\
\text { vida y la salud. }\end{array}$ \\
\hline
\end{tabular}



$\begin{array}{ll}\text { Percepción } & \text { ¿Qué entiende por } \\ \text { actividad física? }\end{array}$

Para usted, ¿qué

Beneficio

Actividad física

Práctica beneficios trae

la práctica de la actividad física?

¿Qué experiencia ha tenido o tiene con respecto a la práctica de la actividad física?
Está relacionada con el movimiento, entendiéndolo como cualquier tipo de movimiento corporal que realiza el ser humano. También se entiende que es un medio para desarrollar competencias interpersonales y sociales necesarias en al ámbito escolar.

La práctica de actividad física en la escuela desempeña un papel muy importante en el desarrollo de competencias individuales e interpersonales, y genera un beneficio para el organismo.

Se observa el desarrollo de prácticas de juego o lúdicas, sobre todo en los docentes de primaria. Se evidencia que el juego es un elemento clave durante la clase, ya que hace parte de su formación como personas; además, estas prácticas van ligadas a la motivación y el disfrute de las clases.

Fuente: elaboración propia.

En la localidad de Suba se observa que, para los docentes, el concepto de educación física es un elemento clave en el currículo escolar, pero también manifiestan que sus alcances son muy limitados, dado que la intensidad de horas en el espacio de las instituciones es insuficiente y es poco el apoyo institucional. No obstante, conciben la educación física como un área de desarrollo que involucra elementos corporales y sociales, elementos que no fueron descritos en la localidad de Ciudad Bolívar. El deporte continúa siendo la principal herramienta para el desarrollo de sus clases, debido a que es la forma más atractiva para que el estudiante realice la práctica. Además, las prácticas deportivas, mencionan los docentes, traen consigo beneficios sociales y no solo físicos o fisiológicos. Por otro lado, los docentes de primaria involucran otras prácticas acordes con la edad, como el juego y las actividades lúdicas, herramientas fundamentales para la formación de los estudiantes. 
Por otra parte, la actividad física es vista por la mayoría como cualquier tipo de movimiento corporal, que permite desarrollar competencias interpersonales y sociales, y que sus beneficios también contribuyen al buen desarrollo biológico.

\section{Categorías emergentes}

En la tabla 5 se observan las categorías emergentes de la localidad Ciudad Bolívar; y en la tabla 6, las de la localidad de Suba. Se aprecia que las subcategorías no presentaron los mismos elementos en las dos localidades, dado que las investigaciones se dieron en contextos distintos. Estas representaciones que manifiestan los docentes de educación física también hacen parte del concepto.

Tabla 5. Categorías emergentes del estudio en Ciudad Bolívar

\begin{tabular}{|c|c|c|}
\hline Categorías & Subcategorías & Localidad de Ciudad Bolívar \\
\hline & $\begin{array}{l}\text { Valor a la } \\
\text { clase de } \\
\text { educación } \\
\text { física }\end{array}$ & $\begin{array}{l}\text { Desde lo planteado por los docentes, el valor agregado a la } \\
\text { educación física es el trabajo corporal. Lo importante que } \\
\text { es empezar a trabajar la educación física desde una tem- } \\
\text { prana edad y hacer un proceso de enriquecimiento motor } \\
\text { que le pueda aportar al desarrollo físico y cognitivo. Se } \\
\text { puede concluir que los docentes valoran la importancia } \\
\text { de la educación física desde dos aspectos fundamentales: } \\
\text { la creación y formación de valores, y el enriquecimiento } \\
\text { motor y adquisición de habilidades corporales. }\end{array}$ \\
\hline Educación física & Idoneidad & $\begin{array}{l}\text { Los docentes mencionan que la clase de educación física } \\
\text { es una herramienta para la iniciación deportiva y el sub- } \\
\text { siguiente rendimiento deportivo, así como para las trans- } \\
\text { formaciones de vida. El maestro se convierte en un guía } \\
\text { para direccionar el rumbo de la vida de los estudiantes, } \\
\text { por esto requiere planeación y preparación de clase, en } \\
\text { lo que debe ser organizado y no estar sujeto improvisa- } \\
\text { ciones. Además, debe tener coherencia entre el discurso } \\
\text { y la práctica, en donde es importante que los estudiantes } \\
\text { observen, de primera mano, buenos comportamientos de } \\
\text { parte del docente en relación con su mismo discurso. }\end{array}$ \\
\hline
\end{tabular}


Sentimientos y valores

\section{Actividad física}

Experiencia

Factores que influyen en la práctica de la actividad física $(\mathrm{AF})$
Aquí, los docentes manifiestan cómo la actividad física hace parte de su vida y cómo influye en su tranquilidad emocional, en la sociabilidad y, en general, en la satisfacción que puede generar la práctica de un deporte o el simple hecho de realizar una caminata. Cabe anotar que los docentes buscan, además, no solo su bienestar mental, sino también el de las personas que los rodean. Algo que es fundamental, es la liberación de las cargas de estrés que se manejan en el día a día.

En general, los docentes de educación física comentan que desde muy pequeños tenían la práctica de la actividad física como una de sus prioridades en la vida. Se menciona el colegio y la universidad como dos de los momentos que más influyeron en la adquisición de hábitos relacionados con la actividad física. Es así como se puede determinar que los docentes de educación física han tenido una vasta experiencia en la práctica de actividad física y tienen el deporte como principal herramienta para este fin.

Los profesores de educación física encuentran que los siguientes factores influyen en la práctica de la AF: la vida familiar, las lesiones, los escenarios deportivos y lúdicos, y los programas encaminados a la actividad física que brinda el Estado.

Fuente: elaboración propia.

En la tabla 5 se evidencian diferentes subcategorías que fueron identificadas por los docentes de Ciudad Bolívar. Estas revelan que la educación física desde una temprana edad permite un proceso de enriquecimiento motor que puede aportar al desarrollo físico y cognitivo, así como a la formación de valores. De igual modo, consideran que en la educación física es clave la idoneidad de los profesores encargados de la clase, pues su formación y desempeño incide directamente en el proceso de crecimiento y desarrollo de sus estudiantes. En relación con la actividad física, los docentes la consideran parte de su cotidianidad, tienen una amplia experiencia en su práctica, a través de la cual buscan su bienestar mental y el de las personas que los rodean. Para los docentes existen algunos factores que pueden influir en la práctica de la actividad física, como lo son la condición económica, los escenarios y los programas académicos. 
Tabla 6. Categorías emergentes del estudio en la localidad de Suba

Categorías Subcategorías Localidad de Ciudad Bolívar

\begin{tabular}{|c|c|c|}
\hline & $\begin{array}{l}\text { Educación } \\
\text { física y salud }\end{array}$ & $\begin{array}{l}\text { Los docentes ven a la educación física como un elemen- } \\
\text { to clave en la salud de los estudiantes, pero consideran } \\
\text { que sus alcances no son suficientes al enfrentar problemas } \\
\text { como la limitación del tiempo de la clase. Esta relación en- } \\
\text { tre educación física y salud, por otro lado, no es explícita } \\
\text { con los estudiantes; es decir, la mayoría de discursos sobre } \\
\text { el tema se centran en la importancia que le ve el maes- } \\
\text { tro a esta asignatura para la salud, pero a los estudiantes } \\
\text { esta importancia no se les recalca o enseña explícitamente } \\
\text { dentro del currículo. Por diferentes motivos, ya sean de } \\
\text { infraestructura o de tiempo de clase, no se presentan con- } \\
\text { ceptos teóricos dirigidos expresamente a este tema. }\end{array}$ \\
\hline \multirow[t]{2}{*}{ Educación física } & $\begin{array}{l}\text { Infraestructura } \\
\text { escolar }\end{array}$ & $\begin{array}{l}\text { En el tema de infraestructura escolar, los docentes re- } \\
\text { fieren que las instalaciones recreativas o deportivas } \\
\text { son insuficientes para la cantidad de alumnos y que } \\
\text { en algunos casos deben desplazarse a parques cercanos } \\
\text { para poder desarrollar las clases. }\end{array}$ \\
\hline & $\begin{array}{l}\text { Condición so- } \\
\text { cioeconómica }\end{array}$ & $\begin{array}{l}\text { La condición socioeconómica que relatan los docentes } \\
\text { acerca de la población con la que trabajan es heterogé- } \\
\text { nea. Se encuentran estudiantes de estratos } 1,2 \text { y } 3 \text {, con } \\
\text { múltiples dificultades familiares, económicas o sociales. } \\
\text { La condición de la familia es la situación problemática } \\
\text { más latente, ya que, según los docentes, la influencia de } \\
\text { la familia es primordial para la educación y desempeño } \\
\text { de los estudiantes, quienes muchas veces no tienen un nú- } \\
\text { cleo familiar estable, lo que afecta el comportamiento y la } \\
\text { autoestima de los niños y jóvenes. Se indicó, por ejemplo, } \\
\text { que existen niños hijos de recicladores, expendedores de } \\
\text { drogas y algunos desplazados por la violencia o por la si- } \\
\text { tuación económica del país. }\end{array}$ \\
\hline
\end{tabular}

Fuente: elaboración propia.

En Suba se aprecia que la práctica y las representaciones sociales frente a la educación física se ven afectadas por varios factores como la infraestructura, la condición social y la inclusión o exclusión del concepto de salud en el marco de la clase. Se relaciona la salud con la educación física como un elemento clave en los estudiantes, ya que esta promueve buenos hábitos para ellos. A su vez, manifiestan que la cantidad de horas no son suficientes para los alumnos, ni los elementos teóricos de salud que son implementados en 
la práctica. Cabe resaltar que las barreras que ellos manifiestan son sociales e individuales, lo que permite afirmar que la actividad física depende del entorno y la actitud de la persona para realizarla.

\section{Conclusiones}

Las representaciones sociales muestran, en común, que la actividad física está relacionada con cualquier movimiento corporal que desarrolla el ser humano, incluyendo las actividades diarias y deportivas. Este concepto no se aleja del propuesto por Rojo (2009), afirma lo siguiente:

La actividad física se define como el movimiento del cuerpo humano producido por la contracción muscular que genera un gasto energético por encima del nivel metabólico de reposo, estando íntimamente relacionada con el ejercicio físico y con el fitness. La actividad física puede tener un motivo laboral, puede referirse a actividades del hogar, a actividades de tiempo libre, transporte, entretenimiento o deportivas. (p. 1)

Los docentes de educación física también manifiestan que al realizar actividad física se obtiene un bienestar físico y emocional. Esto da a entender que quien realiza más actividad física disfruta de estas en un ambiente social y, a su vez, desarrolla competencias individuales e interpersonales, que proporcionan beneficios para el organismo. De esta manera, se observa que los docentes tienen claro que la actividad física influye en el desarrollo integral del ser humano y sus diferentes dimensiones. Con relación a esto, Airasca y Giardini (2009) afirman cómo desde la actividad física se puede integrar una persona fácilmente a un grupo social, hacer parte de él y aportar a este. Esta afirmación no dista de lo que la mayoría de los docentes plasman en sus respuestas. "Así mismo no podemos olvidar que las actividades físicas son prácticas sociales, puesto que las realizan las personas en interacción entre ellas, otros grupos sociales y el entorno" (Airasca y Giardini, 2009).

Por otra parte, la manera en que se práctica la actividad física desempeña un papel muy importante, ya que las herramientas más utilizadas por los docentes en la clase de educación física son el deporte y los juegos lúdicos. Aunque estas metodologías no deberían ser las únicas que se utilizan para incentivar en los alumnos la práctica de la actividad física, vale la pena 
destacar el deporte como principal herramienta; este es el motor que los impulsa y que, en determinados casos, brinda placer. Para la gran mayoría de ellos, la práctica deportiva hoy en día es parte de su vida. Se debe tener en cuenta que las actividades que generan placer están directamente relacionadas con la salud, como lo afirma Tapia y Silva (2016):

Si recordamos que la palabra deporte proviene del latín "disporte" (dis y portare) que etimológicamente significará sustraerse al trabajo, observaremos que el placer está de hecho implícito en esta actividad. Por regla general todo aquello que presupone placer implica salud, aunque debemos admitir algunas extraordinarias excepciones que confirman este lema social. (p. 14)

Se determinó que la educación física es vista como un área integral, en la que se pueden desarrollar múltiples aspectos en el estudiantado, tanto a nivel corporal como a nivel social, reconociendo el cuerpo, la mente, el autocuidado y la salud. Los docentes afirman que el beneficio que le brinda la educación física al ser humano tiene que ver netamente con la adquisición de valores y de hábitos, pero manifiestan que la intensidad de horas en el currículo académico es poco para que sea bien aprovechada. Este concepto, sumado a la utilización del deporte como parte de la enseñanza de la educación física, permite reconocer dos perspectivas diferentes: la primera enfocada a lo social y la segunda hacia lo deportivo. Desde lo social, "la educación física es la educación de la salud, del cuerpo-mente. Se debe enseñar los valores para que el alumno futura personal social, tenga los conocimientos mínimos que le permitan cuidar su cuerpo y mantener su salud" (Solas, 2006, citado por Gualtero y Camargo, 2016). Desde lo deportivo, "la educación física tiene que dar las bases motoras comunes a todos los deportes a fin de que los alumnos si deciden ser deportistas de competición lleguen con unos conocimientos motores básicos a todos los deportes" (Solas, 2006, citado por Gualtero y Camargo, 2016).

Además, se evidenciaron categorías emergentes como la infraestructura. Este es un factor que influye en la práctica de la actividad física y la educación física, ya que en ocasiones no se cuenta con los escenarios propicios para su práctica. Los docentes de educación física también manifestaron que la condición social de la población con la que trabajan es heterogénea, encontrando estudiantes de estratos 1,2 y 3 con múltiples dificultades 
familiares, económicas y sociales. La condición de la familia es la situación problemática más latente; por lo tanto, es uno de los elementos que se debe tener en cuenta al momento de promover la práctica de actividad física.

Se observó que los docentes identificaron barreras para la realización de la actividad física como las políticas institucionales locales y distritales. Por ejemplo, a nivel curricular se les da prioridad a otras áreas del conocimiento, como matemáticas y lenguaje, desplazando poco a poco este espacio académico. Por otra parte, según los docentes, los padres no apoyan las actividades deportivas o recreativas extraescolares por diferentes motivos, por ejemplo, la falta de tiempo de los acudientes para realizar actividad física con sus hijos. Sin embargo, tampoco permiten que los niños salgan solos a los parques a realizar actividades, pues la problemática del sector no posibilita que haya parques seguros para ellos.

Estos hallazgos coinciden con el planteamiento de Márquez, Vives y Garcés de los Fayos (2012) sobre la adherencia a la actividad física, quienes menciona tres factores que son determinantes: los personales, ambientales y programáticos, de los cuales se toma el factor ambiental que es el más nombrado por parte de los docentes entrevistados. Al respecto, estos investigadores afirman que:

[...] los factores situacionales pueden ser tanto aspectos del ejercicio como del ambiente en que se realiza y pueden ayudar o entorpecer la adherencia. Se incluyen aspectos de la vida diaria de las personas, tales como determinados hábitos de vida; aspectos del entorno social como la familia y los compañeros; y también aspectos relacionados con el entorno físico, tales como el clima, los horarios de trabajo y la distancia a la que se encuentran las instalaciones. (Márquez, Vives y Garcés de los Fayos, 2012, p. 230)

La experiencia es una de las características que se resalta para la práctica de la actividad física. Se menciona el colegio y la universidad como dos de los momentos que más influyeron en la adquisición de hábitos relacionados con la actividad física en los docentes. Es así como se puede determinar que los profesores de educación física han tenido una vasta experiencia en la práctica de actividad física y tienen el deporte como principal herramienta para este fin. Esta experiencia influye en las representaciones sociales del docente, así como lo afirma Carretero (1991, citado por Ruiz Ortega, 2009). 


\section{Referencias}

Airasca, D. y Giardini, H. (2009). Actividad física, salud y bienestar. Buenos Aires: Nobuko. Versión Impresa.

Almonacid, A. (2012). La educación física como espacio de transformación social y educativa: perspectivas desde los imaginarios sociales y la ciencia de la motricidad humana. Estudios. Pedagógicos, 38(especial), 177-90. DoI: https://doi. org/10.4067/s0718-07052012000400010

Ariza, J., Laverde, C. y Párraga, O. (2013). Representaciones sociales de la actividad fisica y sus condicionantes en docentes de los ciclos I y II de los colegios distritales de la localidad de los mártires en Bogotá (tesis inédita de pregrado). Universidad Nacional de Colombia. Recuperado de http://bdigital.unal.edu. co/11554/1/561433.2013.pdf

Cerezo, C. (2004). Argumentos sobre la formación inicial de los docentes en educación física. Profesorado, Revista de Currículum y Formación del Profesorado, $8(1), 20$.

Federation Internationale D'Education Physique. (2000). Manifiesto de Educación Física 2000 (traducción: Jorge Díaz Otáñez). Córdoba: Vicepresidencia Mundial para Sudamérica. Recuperado de https://www.medellin.gov.co/irj/go/km/ docs/wpccontent/Sites/Subportal\%20del\%20Ciudadano/Equidad $\% 20 \mathrm{de} \% 20$ Género/Secciones/Plantillas\%20Genéricas/Documentos/2011/Diplomado\%20 PolPub/MANIFIESTO\%20DE\%20EDUCACION\%20FISICA.pdf

Gallo, L. (2010). Los discursos de la educación física contemporánea (versión impresa, fragmentos). Bogotá: Kinesis. Recuperado de http://files.alexaguilaredufisico.webnode.com.co/200000027-1ef3c1f8e0/epilogo \%20Los\%20 discursos \%20de \%20la \%20Educación \%20F\%C3\%ADsica\%20contemporánea \%20LUZ\%20ELENA\%20GALLO\%20(2).pdf

González Arévalo, C., Lleixà Arribas, T., Casamort Ayats, J., Espanya, Ministerio de Educación e Instituto de Formación del Profesorado, I. e I. E. (Espanya). (2010). Educación física: Complementos de formación disciplinar. Barcelona; Madrid: Graó; Ministerio de Educación.

Gualtero, E. y Camargo, D. (2016). Imaginarios Sociales de los profesores de educación física de la UPZ 68 de la localidad de Ciudad Bolivar, referente a la actividad física, la educación física y el cuerpo (tesis de maestría). Universidad Santo Tomás, Bogotá, Colombia. DoI: https://doi.org/10.15332/tg.mae.2017.00401

Leal, E., Aparicio, D., Luti, Y., Acosta, L., Finol, F., Rojas, E., Toledo, A., Cabrera, M., Bermúdez, V. y Velasco, M. (2009). Actividad física y enfermedad 
cardiovascular. Revista latinoamericana de hipertensión, 4(1), 2-17. Recuperado de: https://www.redalyc.org/pdf/1702/170216824002.pdf

Márquez R, Vives L y Garcés de los Fayos, E. (2012). Adherencia y abandono en la actividad física y deportiva. En: R. Marquez, y N. Garatechea (eds.), Actividad Física y Salud (pp. 225-236). Madrid: Ediciones Díaz de Santos.

Maxwell, J. (1996). Un modelo para el diseño de investigaciones cualitativas. Qualitative research design. An Interactive Approach. Londres: Sage Publications. Recuperado de https://www.fceia.unr.edu.ar/geii/maestria/2013/2013_extrasYmetodologia/Palermo_MAXWELL.pdf

Rojo, J. (2009). Un análisis de la actividad física para la salud. Madrid: D - Fundación General de la Universidad Autónoma de Madrid. Recuperado de http://www. encuentros-multidisciplinares.org/Revistan \% C2\%BA14/Jes\%C3\%BAs\%20 Javier\%20Rojo\%20Gonz\%C3\%A1lez.pdf

Rozengardt, R. (2015). La educación física: ciencia, ética, política y pedagogía. Un aporte histórico a la identidad del campo. Perspectivas Metodológicas, 1(1). DOI: https://doi.org/10.18294/pm.2001.560

Ruiz Ortega, L. (2006). Pensamiento docente sobre los procesos de enseñanza en la Educación Física. Revista Latinoamericana de Estudios Educativos, 2(1), 79-99. Recuperado de https://www.redalyc.org/pdf/1341/134116859004.pdf

Secretaría de Cultura Recreación y Deporte. (2015). Agenda Pública Local. Deporte, Recración, Actividad Física, Educación Física y Equipamientos. Agenda Local Suba. Universidad Distrital- Alcaldía Mayor de Bogotá. Recuperado de http:// www.culturarecreacionydeporte.gov.co/sites/default/files/agenda_publica_local_de_suba_1.pdf

Secretaría de Cultura Recreación y Deporte (2015). Agenda Pública Local. Deporte, Recración, Actividad Física, Educación Física y Equipamientos. Agenda Local Ciudad Bolivar. Universidad Distrital- Alcaldía Mayor de Bogotá. Recuperado de: https://www.culturarecreacionydeporte.gov.co/sites/default/files/agenda_ publica_local_de_ciudad_bolivar_1.pdf

Tapia, E. (2016). Guía de ejercicios básicos específicos para contribuir a la iniciación en la natación de los estudiantes de 6 to y 7 mo año de educación básica de la Cooperativa Juan Montalvo en Guayaquil. Recuperado de http://repositorio.ug.edu.ec/handle/redug/27833

Toscano, G. (2011). La utilización del método comparativo en estudios cualitativos en Ciencia Política y Ciencias Sociales: diseño y desarrollo de una tesis doctoral. Kairos: Revista de temas sociales, (27), 7. 
